\title{
Distinction between Thermal and Stress-Induced Martensitic Transformations and Inhomogeneity in Internal Stress
}

\author{
Hidekazu Sakamoto \\ Department of Aerospace Engineering, School of Science and Engineering, Teikyo University, Utsunomiya 320-8551, Japan
}

\begin{abstract}
A concept of inhomogeneous internal stress called shape change stress (SCS) was introduced in martensitic transformations (MTs). The SCS was considered to be required because martensite plates formed on cooling must deform the surrounding untransformed parent. The concept enabled successful elucidation of the reason for the progress of thermally induced MTs (TIMTs) with changing temperature and for that of stress-induced MTs (SIMTs) under constant stress on the basis of the Gibbs phase rule. Decreasing SCS with decreasing specimen size (mass) explained the downward shifts of experimental equilibrium temperatures $T_{o}^{*}=\left(M_{s}+A_{f}\right) / 2$ with decreasing specimen mass $m$ so far observed in the TIMTs of some shape memory alloys. Furthermore, the concept that no SCS is generated either in SIMTs or at the ultimate end of $m=0$ in TIMTs rationalized the following observation on a monocrystalline $\mathrm{Cu}-13.4 \mathrm{Al}-4.2 \mathrm{Ni}$ (mass\%) alloy. That is, the variations of $M_{s}$ and $M_{f}$ with $m$ converged at the extrapolation to $m=0$, and those of $A_{s}$ and $A_{f}$ also did; $M_{s}^{\#}=M_{f}^{\#}$ and $A_{s}^{\#}=A_{f}^{\#}$ (the superscript \# indicates the transformation temperatures at the ultimate end of $m=0)$. The former coincided with $T^{L}(0)$ where the dependence of transformation stress $\sigma^{L}$ on temperature was extrapolated to stress zero, and the latter coincided with $T^{U}(0)$ where a similar extrapolation in the case of reverse transformation stress $\sigma^{U}$ was performed.
\end{abstract}

(Received February 28, 2002; Accepted July 10, 2002)

Keywords: shape memory alloys, transformation temperatures, transformation stress, experimental equilibrium temperatures, Gibbs phase rule, shape change stress, elastic back stress, specimen size effect

\section{Introduction}

Martensitic transformations (MTs) are induced not only by cooling some kinds of alloys below the MT start temperature $M_{s}$ but also by the application of external stress above $M_{s}$. Upon comparing the characteristics of thermally induced MTs (TIMTs) with those of stress-induced MTs (SIMTs), it is evident that a paradox arises between them, which is described as follows.

The progress of TIMTs occurs with changing temperature; transformations on cooling proceed in the temperature range from $M_{s}$ to $M_{f}$, and their reverse transformations on heating proceed from $A_{s}$ to $A_{f}$. That is, the amount of thermally induced martensite is a function of temperature. Usually, these temperature ranges where transformations and their reversions take place are not small but are somewhat extended, for example, from several degrees to some tens of degrees in thermoelastic MTs.

In contrast, SIMTs in monocrystalline specimens ${ }^{1,2)}$ and even in polycrystalline ones in a $\mathrm{Ti}-\mathrm{Ni}$ alloy system ${ }^{3)}$ progress in a different manner from TIMTs. That is, transformations on loading proceed under an almost constant stress $\sigma^{L}$ and their reverse transformations on unloading also proceed under a different almost constant stress $\sigma^{U}$. Stress-strain (s-s) curves show almost plateau regions during transformations and their reversions; that is, stress ranges where transformations and their reversions take place are rather small. In other words, the MT start stress on loading, $\sigma_{s}^{L}$, is equal or nearly equal to the MT finish stress, $\sigma_{f}^{L}$, and the case for reverse transformation start and finish stresses is almost the same. That is, the amount of stress-induced martensite is a function of strain.

The contrast between TIMTs and SIMTs described above is clearly seen in Fig. 1. It shows a differential scanning calorimetry (DSC) curve associated with the TIMT of a spec-
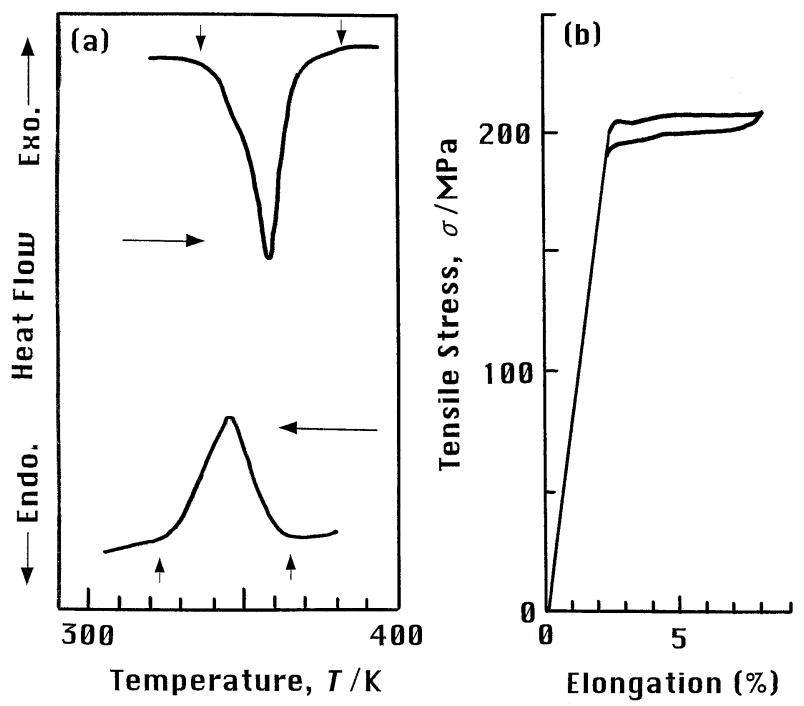

Fig. 1 Diagram showing the contrast between TIMTs and SIMTs in a $\mathrm{Cu}-13.4 \mathrm{Al}-4.2 \mathrm{Ni}$ (mass\%) alloy single crystal; (a) a DSC curve of a specimen with mass of $101.5 \mathrm{mg}$, and (b) a tensile s-s curve at $453 \mathrm{~K}$.

imen with mass of $101.5 \mathrm{mg}$, (a), and a tensile s-s curve associated with the SIMT at $453 \mathrm{~K}$, (b), of a $\mathrm{Cu}-13.4 \mathrm{Al}-4.2 \mathrm{Ni}$ (mass\%) alloy single crystal. ${ }^{4,5}$ )

Obviously, small stress ranges of transformations in SIMTs make a sharp contrast with somewhat extended temperature ranges of transformations in TIMTs. These phenomena are known to most of researchers in this field, but it appears that no one has discovered any problems. Therefore, the origin of the differing behaviors between TIMTs and SIMTs has not been elucidated yet.

Another distinction is also observed between them. That is, in TIMTs, specimens remain almost unchanged in terms of their shape as a whole. Meanwhile, in SIMTs, macroscopic 
shape changes of specimens, such as elongation under tensile stress, take place.

Incidentally, in SIMTs, critical stress for inducing a martensite phase on loading, $\sigma^{L}$, linearly increases with increasing test temperature. The Clausius-Clapeyron equation well explains the linear dependence of $\sigma^{L}$ on temperature, $\mathrm{d} \sigma^{L} / \mathrm{d} T$. Here, let $T^{L}(0)$ represent the temperature where $\mathrm{d} \sigma^{L} / \mathrm{d} T$ is extrapolated to stress zero. The temperature $T^{L}(0)$ has been reported to agree with $M_{s}$ observed when tensile specimens were cooled in some shape memory alloys. ${ }^{2,6-8)}$ This agreement intuitively appears to be plausible to almost all researchers in this field; therefore, the agreement has been accepted as correct. Upon considering the matter carefully, however, it will be found that the agreement is doubtful. The following argument elucidates the problem.

First, let us presuppose that $M_{s}$ agrees with the temperature $T_{s}^{L}(0)$ where the dependence of $\sigma_{s}^{L}$ on temperature, $\mathrm{d} \sigma_{s}^{L} / \mathrm{d} T$, is extrapolated to stress zero. If this presupposition is valid, it is also valid to assume that $M_{f}$ agrees with the temperature $T_{f}^{L}(0)$ where the dependence of $\sigma_{f}^{L}$ on temperature, $\mathrm{d} \sigma_{f}^{L} / \mathrm{d} T$, is extrapolated to stress zero. This assumption, however, creates the following paradox.

The temperature $T_{s}^{L}(0)$ must also be equal or nearly equal to $T_{f}^{L}(0)$, because $\sigma_{s}^{L}$ is equal or nearly equal to $\sigma_{f}^{L}$. Hence, it follows naturally that $M_{s}$ must be equal or nearly equal to $M_{f}$. Clearly, this deduction is incompatible with the observation that in TIMTs $M_{s}$ is higher than $M_{f}$ by several degrees to some tens of degrees. This incompatibility indicates that the assumption is incorrect, that is, $M_{f}$ disagrees with $T_{f}^{L}(0)$.

On the other hand, as for the reverse transformations, the circumstances are somewhat different. Let $T_{s}^{U}(0)$ and $T_{f}^{U}(0)$ represent the temperatures where the dependence of reverse transformation start stress $\sigma_{s}^{U}$ on temperature, $\mathrm{d} \sigma_{s}^{U} / \mathrm{d} T$, and that of finish stress $\sigma_{f}^{U}$ on temperature, $\mathrm{d} \sigma_{f}^{U} / \mathrm{d} T$, respectively, are extrapolated to stress zero. It has not been recognized yet that $A_{s}$ agrees with $T_{s}^{U}(0)$ and $A_{f}$ agrees with $T_{f}^{U}(0)$.

Under these circumstances, it follows that almost all researchers in this field have some doubt on the agreement realized only between $M_{s}$ and $T_{s}^{L}(0)$. The paradox is diagrammatically illustrated in Fig. 2. The paradox is a fundamental problem for MTs; it should not be ignored. If the problem remains unsolved, the correspondence between the behavior of TIMTs and that of SIMTs will not be able to be strictly discussed.

Therefore, one purpose of the present paper is to resolve this issue. The other is to explain the reason for the progress of SIMTs at an almost constant stress and for that of TIMTs with changing temperature. Both purposes have been successfully achieved by introducing a concept of internal stresses called shape change stress and elastic back stress generated during the formation of martensite plates in TIMTs.

\section{Concept of Shape Change Stress}

The concepts of shape change stress and elastic back stress introduced to resolve the above issue are explained below. These stresses are generated when a martensite plate is formed on cooling. First, the key to the resolution is outlined. For the martensite plate to form in TIMTs, the generation of an internal stress called shape change stress is re-

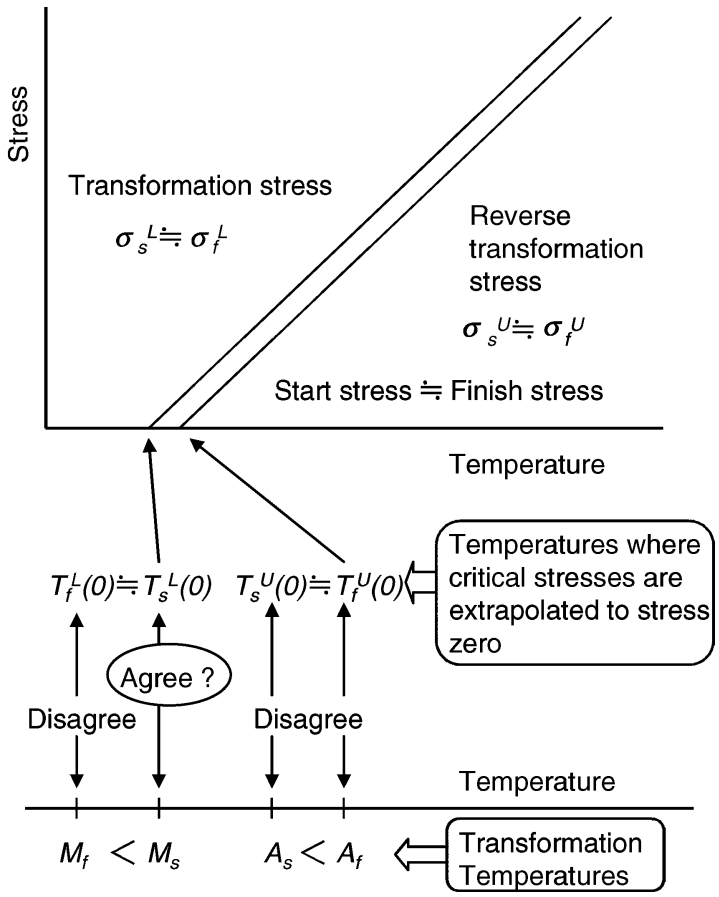

Fig. 2 Diagram illustrating the doubt on the agreement of $M_{s}, M_{f}, A_{s}$ and $A_{f}$ with $T_{s}^{L}(0), T_{f}^{L}(0), T_{s}^{U}(0)$ and $T_{f}^{U}(0)$, respectively.

In thermally induced MTs, the transforming region must generate stress to deform the surrounding parent.

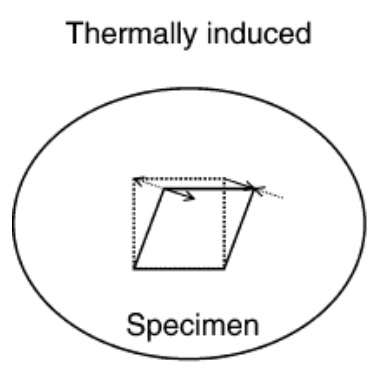

Stress-induced

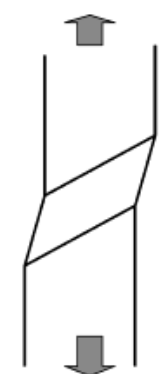

Shape change of a martensite plate is caused by

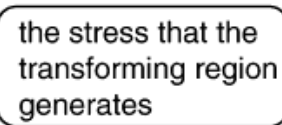

the effect of externally applied stress

Fig. 3 Schematic illustration showing that the generation of shape change stress associated with the formation of a martensite plate is necessary in TIMTs but unnecessary in SIMTs.

quired, because the plate must elastically deform the surrounding untransformed parent to cause the shape change. On the other hand, in SIMTs, no generation of such internal stress is required at all, because the shape change of a stress-induced martensite (SIM) plate is caused by the applied external stress. Figure 3 schematically illustrates these two situations. The details are described in the following.

In TIMTs, shape change has to take place in a certain region about to transform into a martensite plate without the aid of external stress. For the plate to form, the region itself has to generate stress to deform the surrounding untransformed parent elastically. Usually, a self-accommodation mechanism operates to minimize net shape change by arranging some 


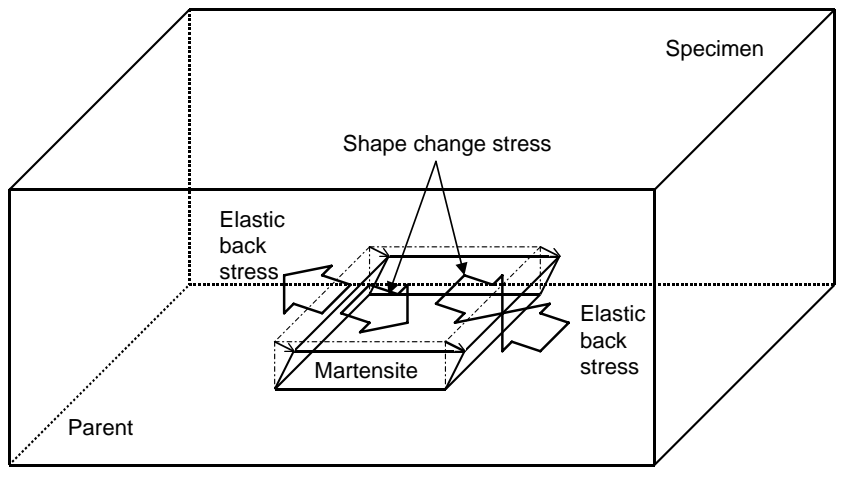

Fig. 4 Three-dimensional schematic illustration showing the generation of shape change stress and elastic back stress associated with the formation of a martensite plate in TIMTs.

variants of martensite plates. Perfect self-accommodation by which no net shape change occurs at all is, however, impossible. Moreover, each variant itself must cause its own shape change, even though the self-accommodation mechanism operates. Thus, whether the mechanism operates or not, each variant must generate stress, or else no transformation can occur.

The generated stress will henceforth be called shape change stress. The surrounding parent is subjected to the shape change stress from the martensite plate or the selfaccommodation group of the martensite plates. Little attention has been paid to such shape change stress generated during forward transformations on cooling. On the other hand, it is already known that shape recovery stress is generated when deformed martensites reverse transform into parents on heating. The shape change stress generated on cooling developed here corresponds to the shape recovery stress generated on heating. Thus, the concept of shape change stress is relevant.

Granted that such shape change stress is generated, an elastic back stress or constraint effect against the martensite plate or a group of plates must be generated as the reaction from the surrounding parent region. In other words, the plate or group of plates is subjected to the elastic back stress. Thus, the formation of martensite plates involves the generation of internal stresses such as the shape change stress and the elastic back stress. The situation is schematically illustrated two-dimensionally on the left-hand side in Fig. 3 and threedimensionally in Fig. 4. Hereafter, the shape change stress and the elastic back stress are abbreviated to SCS and EBS, respectively.

These internal stresses, SCS and EBS, generated are inferred to increase with increasing specimen size, because a large stress will be necessary to deform the surrounding untransformed parent of a large mass. Furthermore, they are also expected to increase with increasing size of a martensite plate formed because of its large shape change. That is, they are not intrinsic to a substance but dependent essentially on both specimen size and martensite plate size.

Here, the specimen size concerned will be around millimeter scale. The martensite plate size also plays an important role on the magnitude of the internal stress field. Decreasing plate size reduces the region where stress field is generated. It is expected that large stress field is generated over the region at least from several times to several tens of times of a martensite plate size. For example, as for $\beta_{1}^{\prime}$ martensite in $\mathrm{Cu}$-based shape memory alloys, the plate size is $\sim 0.1 \mathrm{~mm}$. Therefore, the region where a stress field is generated will be on the millimeter scale.

Incidentally, the magnitude of SCS in parents and that of EBS in martensites are presumed to be inhomogeneous. That is, their magnitudes are expected to vary according to the position in the specimen.

The SCS and EBS developed here are similar to thermal stress generated when a substance is heated with both ends being fixed. Assuming that the substance is heated with both ends or one end free, no constraint and therefore no thermal stress will be generated at all. Likewise, assuming that no untransformed parent surrounds a martensite plate, the shape change of the plate takes place freely. Therefore, neither SCS nor EBS will be generated at all. This similarity of SCS and EBS to the thermal stress is because the shape strain associated with MTs is eigen strain, that is, stress-free strain, as well as dilatation strain.

On the other hand, SIMTs in single crystal specimens present a striking contrast to TIMTs. External stress applied to a specimen causes shear-type movement of atoms associated with the formation of a martensite plate of a single variant. The single variant has the maximum Schmid factor or gives rise to the maximum elongation under tensile loading. ${ }^{9)}$ The SIM plate traverses the specimen, as schematically shown on the right-hand side in Fig. 3. That is, the SIM plate is not subjected to any EBS from the neighboring untransformed parent. In other words, the neighboring parent is not subjected to any SCS but to the same external stress as the SIM plate. On further loading, therefore, the parent is also subsequently going to transform into the same variant as that of the SIM plate first formed by the external stress. That is, no generation of SCS is required at all to form the SIM plate. Thus, a clear distinction is present in SCS between TIMTs and SIMTs.

\section{Application of Gibbs Phase Rule to MTs}

Introducing the concept of the SCS and EBS enables us to explain the reason for the progress of SIMTs at an almost fixed stress and for that of TIMTs with changing temperature. The reason will be explained on the basis of the Gibbs phase rule. It states that if a system containing $C$ components and $P$ phases is in equilibrium, the number of degrees of freedom $F$ is given by

$$
P+F=C+2 .
$$

In MTs, $C$ is considered to be one, because no compositional change occurs. Then, it follows that $P+F=3$. In a state where a parent and a martensite coexist, $P=2$. Therefore, $F=1$; the system has one degree of freedom. Accordingly, only one intensive variable, either temperature or stress, can be varied.

For MTs, both TIMTs and SIMTs, hysteresis is involved between transformations and the reverse transformations. The hysteresis is unavoidable, because MTs are displacive transformations in solids. That is, frictional resistance is inevitable against shear-type displacement of atoms associated with the formation and reversion of a martensite plate and must be 
overcome by supercooling and superheating, respectively. ${ }^{10)}$ Therefore, MTs are, in general, irreversible processes. However, at least in shape memory alloys, a martesite phase perfectly recovers its original parent phase by the reverse transformation. Although reversible processes do not actually occur, it is useful to imagine a hypothetical reversible process, because thermodynamical analysis will be simplified. Then, let us assume an idealized frictionless and quasistatic thermoelastic MT and apply the phase rule to the idealized thermoelastic MT.

Here, the condition of one degree of freedom in the case of coexistence of two phases will be applied to SIMTs and TIMTs. In SIMTs, one degree of freedom indicates that when the test temperature is specified, stress where the two phases coexist in equilibrium is also specified. Accordingly, if the stress where the two phases coexist changes, this contradicts the phase rule.

Incidentally, if an SIMT takes place at a fixed temperature, the transformation on loading proceeds at an almost constant stress $\sigma^{L}$ and the reverse transformation on unloading proceeds at another almost constant stress $\sigma^{U}$. Figure 1(b) shows an example of such s-s curves. The progress of the SIMT at a fixed stress is exactly in agreement with the phase rule. In addition, the behavior indicates that the stress field generated inside the single crystal specimen by the external stress is homogeneous throughout.

The plateau regions in the present s-s curve are, however, somewhat uneven. This is probably because the uniformity of the cross-sectional area through the gauge length portion of the tensile specimen was not altogether satisfactory. The plateau will become more even with improving the uniformity of the cross-sectional area. Indeed, in a previous investigation on a $\mathrm{Cu}-14.0 \mathrm{Al}-4.2 \mathrm{Ni}$ (mass\%) alloy single crystal, ${ }^{1)}$ $\mathrm{s}$-s curves showing more even plateaus than the present one have been obtained. This is because the degree of parallelism of the opposite surfaces on the gage length portion of the tensile specimen used was carefully adjusted.

On the other hand, in TIMTs, the externally applied stress is specified to be zero. Therefore, in a state maintaining the coexistence of the two phases, the number of degrees of freedom is also calculated to be one. Therefore, in a thermal cycle under no external stress, the temperature where the two phases coexist in equilibrium is also specified. Accordingly, the coexistence of the two phases should proceed under a constant temperature.

In reality, changing temperature is, however, necessary for the progress of TIMTs, as seen in Fig. 1(a). The amount of martensite increases with decreasing temperature. The reason is that the untransformed parent phase is subjected to the inhomogeneous internal stress field of SCS from martensite plates already formed. That is, the magnitude of the SCS in the untransformed parent region varies depending on each position in the parent. Therefore, the transformation temperature at each position alters correspondingly. Hence, the progress of TIMTs by changing temperature is a phenomenon that obeys the Gibbs phase rule.

Nevertheless, TIMTs can progress even at a fixed temperature in the following situation. Salzbrenner and Cohen have reported a rectangular hysteresis loop in a \% martensite versus temperature diagram in a single interface transformation in a single crystal of a $\mathrm{Cu}-14 \mathrm{Al}-2.5 \mathrm{Ni}$ (mass\%) alloy. ${ }^{11)}$ That is, the forward transformation has started at a temperature $T_{g}$ and has finished at the same temperature, and the reverse transformation has started at another temperature $T_{r}$ and has finished at the same temperature. The reason is again explained on the basis of the Gibbs phase rule as follows. In single interface transformations, a martensite plate formed traverses the specimen. Hence, the shape change of the plate takes place without any constraint. The situation is similar to that in SIMTs in single crystals. Therefore, the untransformed parent is never subjected to SCS from the neighboring martensite plate. Accordingly, it follows that single interface transformations proceed at a fixed temperature.

Incidentally, the specimen will show a large macroscopic shape change in the single interface transformation, as well as in SIMTs. Therefore, it is reasonable to state the following. When transformations proceed at a fixed temperature or stress, no SCS is generated during the formation of a martensite plate, and the specimen undergoes a large shape change.

Here, transformation behavior in a thermal cycle under constant load is referred to. If a single crystal specimen is subjected to a thermal cycle under constant load, each of transformation and reversion is expected to take place at a fixed temperature, because stress field is homogeneous throughout the specimen. However, no experiment has been so far performed on single crystals, because this kind of test is important from the practical point of view. Therefore, transformation behavior under a constant load has been investigated only on polycrystalline specimens. For example, the following points have been clarified for Ti-Ni alloy systems.

In a polycrystalline $\mathrm{Ti}-50.2 \mathrm{at} \% \mathrm{Ni}$ alloy, the ranges of transformation temperatures for bulk specimens have been found to be larger than that for wire specimens because of a large geometrical constraint effect in the transverse direction in the bulk. ${ }^{12,13)}$ In SIMTs of polycrystalline specimens, shape change of a martensite plate in a grain is subjected to constraint from the surrounding grains. Therefore, SCS and EBS are generated, different from single crystals. In a polycrystalline $50 \mathrm{Ti}-40 \mathrm{Ni}-10 \mathrm{Cu}(\mathrm{at} \%)$ alloy, transformation behaviors of bulk material fabricated through conventional melting and of rapidly solidified thin foils have been investigated under constant load. ${ }^{14)}$ As a result, the following points have been revealed. The ranges of transformation temperatures in the thin foils are narrower than those in the bulk, and transformation strain in the former is larger than that in the latter. These results also substantiate the validity of the concept of SCS and EBS developed here.

\section{Effect of SCS on Characteristics of TIMTs Through Specimen Size}

The validity of the concept of SCS has been thus well proved by the successful elucidation of the contrast in transformation behavior between TIMTs and SIMTs. Let us now return to the doubt on the agreement between $M_{s}$ and $T_{s}^{L}(0)$. The problem is also resolved by taking the effect of SCS on the behavior of TIMTs into consideration. That is, the magnitude of SCS generated inside a specimen is expected to increase with increasing specimen size. Therefore, one can predict that this increase in the magnitude of SCS with 
increasing specimen size will alter transformation temperatures in TIMTs with specimen size. In fact, the present author and coworkers have recently found through DSC measurement that the characteristics of thermoelastic MTs in some shape memory alloys have altered with specimen size (mass). ${ }^{4,5,15-17)}$ Here, the specimen size concerned is of millimeter scale. No other researcher has systematically investigated the effect of specimen size on transformation behavior.

The effect of specimen mass on transformation temperatures has thus far been investigated for transformations between B2 parent and B19' monoclinic martensite in a polycrystalline $\mathrm{Ti}-50 \mathrm{~mol} \% \mathrm{Ni}$ alloy, ${ }^{15,16)}$ between $\mathrm{B} 2$ parent and B19 orthorhombic martensite in a polycrystalline $49.5 \mathrm{Ti}-$ $40 \mathrm{Ni}-10 \mathrm{Cu}-0.5 \mathrm{Al}(\mathrm{mol} \%)$ alloy, ${ }^{17)}$ between $\beta_{1}$ parent $\left(\mathrm{DO}_{3}-\right.$ type ordered structure) and $\beta_{1}^{\prime}$ martensite $\left(18 \mathrm{R}_{1}\right.$-type longperiod stacking-order [LPSO] structure in Ramsdell notation) in a single crystal of a $\mathrm{Cu}-13.4 \mathrm{Al}-4.2 \mathrm{Ni}$ (mass\%) alloy, ${ }^{4,5)}$ and so forth. As a result, in any of these alloys, TIMT characteristics have been found to alter with specimen mass as follows.

With decreasing specimen mass, $M_{s}$ and $A_{f}$ have shifted to lower temperatures logarithmically, while $M_{f}$ and $A_{s}$ have shifted to higher temperatures. Consequently, these shifts have effected the following two alterations. First, experimental equilibrium temperatures $T_{o}^{*}=\left(M_{s}+A_{f}\right) / 2$ have shifted to lower temperatures. Second, the temperature ranges where the transformations and the reverse transformations take place, from $M_{s}$ to $M_{f}$ and from $A_{s}$ to $A_{f}$, respectively, have decreased.

Furthermore, the observed variations of transformation temperatures with specimen mass have been extrapolated to specimen mass zero. As a result, it has been revealed that transformation start and finish temperatures on cooling have converged to coincide at specimen mass zero; that is, $M_{s}^{\#}=$ $M_{f}^{\#}$, and for reverse transformation ones on heating it is likewise; that is, $A_{s}^{\#}=A_{f}^{\#}$ (the superscript \# indicates the transformation temperatures at the ultimate end of specimen mass zero). Here, the situation at or near the ultimate end of specimen mass zero is such that a martensite plate traverses a thin planar (two dimensional) or thin rod (one dimensional) single crystal.

Incidentally, transformation temperatures will shift also with scanning rate $\mathrm{d} T / \mathrm{d} t$, especially in a large specimen, probably because of inhomogeneity in temperature. As for $\mathrm{Ti}-50 \mathrm{~mol} \% \mathrm{Ni}^{16)}$ and $49.5 \mathrm{Ti}-40 \mathrm{Ni}-10 \mathrm{Cu}-0.5 \mathrm{Al}(\mathrm{mol} \%)^{17)}$ alloys, transformation temperatures of specimens of various mass have been already investigated as a function of scanning rate. By extrapolating the obtained $\mathrm{d} T / \mathrm{d} t$ dependence of the MT characteristics to $\mathrm{d} T / \mathrm{d} t=0$, the one under infinitesimally slow $\mathrm{d} T / \mathrm{d} t$, that is, under quasistatic condition (QSC), has been deduced.

As a result, the following has been found. The MT characteristics under QSC have shown specimen mass dependence, as well as those measured at a finite $\mathrm{d} T / \mathrm{d} t$, although the dependence is smaller than that measured at a finite $\mathrm{d} T / \mathrm{d} t$. With decreasing specimen mass, $T_{o}^{*}$ under QSC, $T_{o}^{* Q S C}$, has shifted to lower temperatures and transformation temperature ranges under QSC, from $M_{s}^{Q S C}$ to $M_{f}^{Q S C}$, and from $A_{s}^{Q S C}$ to $A_{f}^{Q S C}$, have decreased to zero. Besides, $M_{s}^{\#}=M_{f}^{\#}$ and
$A_{s}^{\#}=A_{f}^{\#}$ have remained unchanged irrespective of scanning rate. These results indicate that, under QSC, the amount of thermally induced martensite in a specimen of a finite mass is a function of temperature, and that the pure effect of SCS and EBS on transformation temperatures can be derived. Very recently, similar results have been obtained on the $\beta_{1} \Leftrightarrow \beta_{1}^{\prime}$ transformations in a $\mathrm{Cu}-13.8 \mathrm{Al}-4.2 \mathrm{Ni}$ (mass\%) alloy single crystal.

Moreover, in the single crystal of the $\mathrm{Cu}-13.4 \mathrm{Al}-4.2 \mathrm{Ni}$ alloy, the behavior of $\beta_{1} \Leftrightarrow \beta_{1}^{\prime}$ transformation psuedoelasticity has also been investigated as a function of temperature above $M_{s}$. As a result, the former $M_{s}^{\#}=M_{f}^{\#}$ has been found to coincide also with $T^{L}(0)$ and the latter $A_{s}^{\#}=A_{f}^{\#}$ with $T^{U}(0){ }^{4,5)}$ The observed coincidence is shown in Fig. 5. It is composed of two diagrams; one illustrates the variations of $\sigma^{L}$ and $\sigma^{U}$ with temperature and the other shows the variations of transformation temperatures measured at $\mathrm{d} T / \mathrm{d} t= \pm 8.3 \times 10^{-2} \mathrm{~K} / \mathrm{s}$ with specimen mass. To clarify the coincidence, the part around $\sigma^{L}=\sigma^{U}=0$ and specimen mass zero in Fig. 5 is magnified and schematically illustrated in Fig. 6.

The origin of the coincidence is explained as follows. It is reasonable to infer that no SCS is required at all at the ultimate end of the specimen size zero in TIMTs. The same situation that no SCS is required stands also in the case of SIMTs, as described in Chapter 2. This rationalizes the observed coincidence: $M_{s}^{\#}=M_{f}^{\#}=T^{L}(0)$ and $A_{s}^{\#}=A_{f}^{\#}=T^{U}(0)$. From these results, it can be stated that $M_{s}$ of a specimen of a finite specimen size cannot agree with $T_{s}^{L}(0)$. Thus, the finding of the phenomenon that the characteristics of TIMTs are altered with specimen mass has brought the paradox set up in Chapter 1 to a settlement. In addition, these results also substantiate the validity of the concept of SCS.

Incidentally, many researchers may have some doubt about the accuracy of transformation temperatures determined through DSC measurement where the temperature

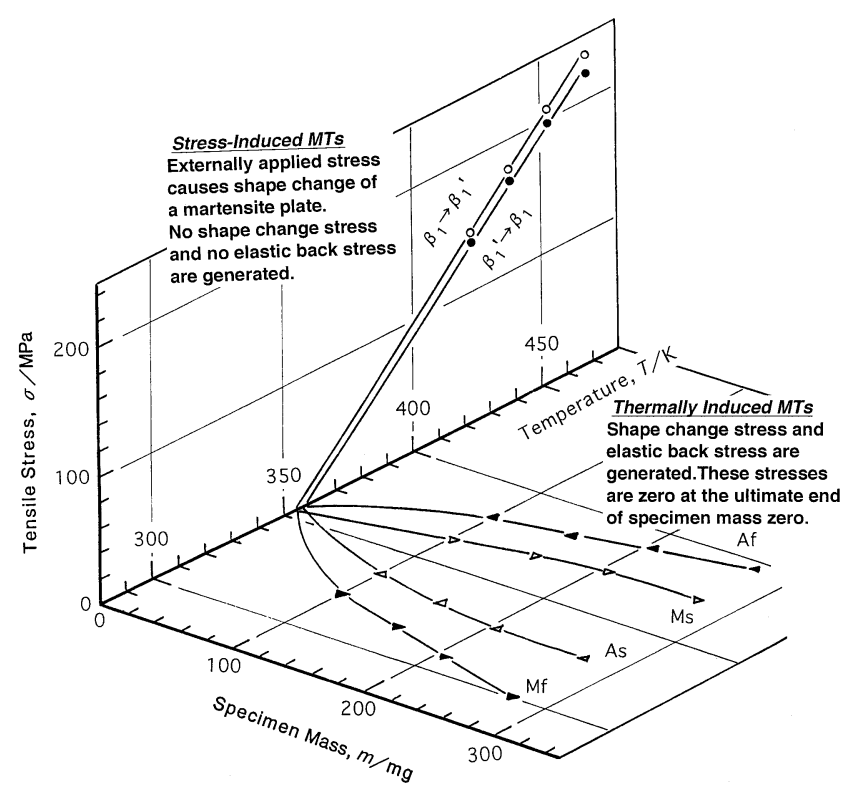

Fig. 5 Combined diagram showing the coincidence between $M_{s}^{\#}=M_{f}^{\#}$ and $T^{L}(0)$ and that between $A_{s}^{\#}=A_{f}^{\#}$ and $T^{U}(0)$ in a single crystal of the $\mathrm{Cu}-13.4 \mathrm{Al}-4.2 \mathrm{Ni}$ alloy. 


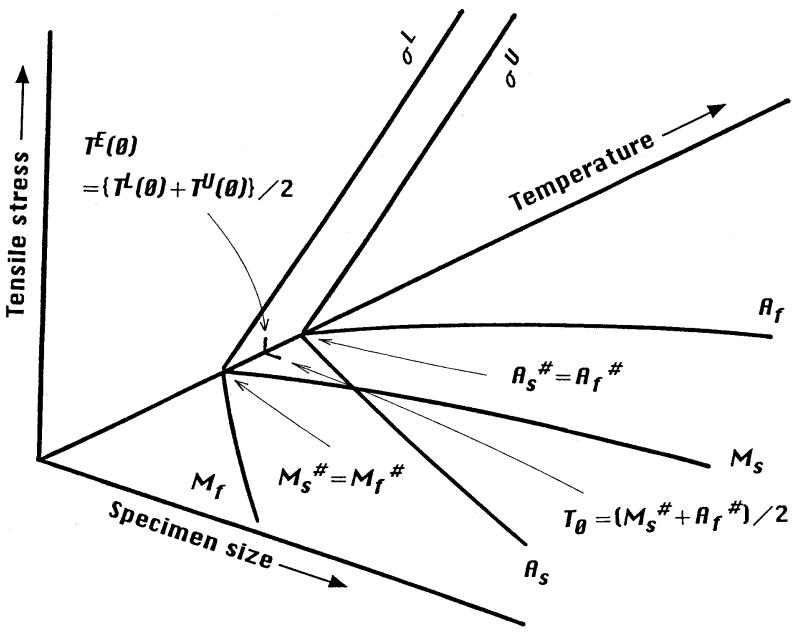

Fig. 6 Enlarged schematic diagram around $\sigma^{L}=\sigma^{U}=0$ and specimen mass zero in Fig. 5.

measured with a thermocouple is the one outside a specimen cell. Therefore, there may be some gap between the measured temperature $T_{s m}$ and the temperature of a specimen $T_{s}$, especially in the case of a large size specimen and a high scanning rate. Strictly speaking, detailed analysis on the gap is necessary. Besides, detailed investigation is also required on the transformation temperatures of specimens of smaller mass than $100 \mathrm{mg}$, although the specimen preparation is difficult.

Nevertheless, it is not unreasonable to suppose the followings. The gap is thought to reduce with decreasing specimen mass and with decreasing scanning rate. In other words, the accuracy of transformation temperatures is expected to increase with decreasing specimen mass. As a result, the extrapolated temperatures to specimen mass zero are expected to remain unchanged. Therefore, it is safe to state that the coincidence between $M_{s}^{\#}=M_{f}^{\#}$ and $T^{L}(0)$ and between $A_{s}^{\#}=A_{f}^{\#}$ and $T^{U}(0)$ in Fig. 5 is not entirely erroneous. Paradoxically, if no coincidence exists between them, we have to discover some proper reason for the disagreement.

Consequently, the idea that $T_{s}^{L}(0)$ agrees with $M_{s}$ of the tensile specimen is seen to be incorrect. This is also confirmed by the following discussion. The effect of specimen size on transformation temperatures described above is clearly observed in TIMTs of tensile specimens. Hence, $M_{s}$ of tensile specimens will shift with specimen size. Meanwhile, no specimen size effect is expected to be observed in SIMTs, because the parent region neighboring SIM plates is subjected to no SCS as previously stated; $T^{L}(0)$ is considered to remain unaltered, irrespective of tensile specimen size. Therefore, as an inevitable consequence, one deduces that $T^{L}(0)$ remaining unaltered cannot agree with $M_{s}$ shifting with specimen size.

Now, the observed variations of experimental equilibrium temperatures $T_{o}^{*}$ with specimen mass are discussed in further detail. In the $\mathrm{Cu}-13.4 \mathrm{Al}-4.2 \mathrm{Ni}$ alloy shown in Fig. 5, $T_{o}^{*}$ shifts around $30 \mathrm{~K}$ to higher temperatures with the increase of specimen mass to $300 \mathrm{mg}$. On the basis of the above discussion, one can correctly presume that the variations of $T_{o}^{*}$ with specimen mass are also attributed to the effect of SCS varying with specimen mass. The variations of $T_{o}^{*}$ have been, however, thus far elucidated to be caused by nonchemical energy $G_{\text {nonc* }}$ generated when martensite plates form at $M_{s}{ }^{10,15)}$
Here, the subscript $*$ is appended to denote energy generated when a fraction $f_{o}$ of martensite plates form at $M_{s}$. The present author and coworkers have already derived an equation $T_{o}^{*}=\left(\Delta H^{P \rightarrow M}+\Delta G_{\text {nonc* }}^{P \rightarrow M}\right) / \Delta S^{P \rightarrow M}$ which gives the variations of $T_{o}^{*}$ with $\Delta G_{\text {nonc* }}^{P \rightarrow{ }^{10}}{ }^{10}$ Here, $\Delta H^{P \rightarrow M}$ is the enthalpy change associated with a transformation and $\Delta S^{P \rightarrow M}$ entropy change, and $\Delta G_{\text {nonc* }}^{P \rightarrow M}$ is the difference in $G_{n o n c *}$ between a martensite and a parent; that is, $G_{\text {nonc* }}^{M}-G_{\text {nonc* }}^{P}$.

The nonchemical energy $G_{n o n c *}$ concerned is the sum of elastic energy $E_{\text {elas* }}$ and interfacial energy $E_{\text {face* }}$. As for $E_{\text {elas* }}$, it is recognized on the basis of the discussion in Chapter 2 that the generation of the elastic energy in parents $E_{\text {elas* }}^{P}$ is ascribed to SCS developed and that in martensites $E_{\text {elas* }}^{M}$, to EBS. If $E_{\text {elas* }}$ is much larger than $E_{\text {face* }}, G_{\text {nonc* }}$ is governed by the former. It has been previously shown ${ }^{11)}$ that, with increasing specimen mass, $E_{\text {elas* }}^{P}$ becomes much larger than $E_{\text {elas* }}^{M}$. In this situation, it must have, therefore, become apparent by now that the observed upward shifts of experimental equilibrium temperature $T_{o}^{*}$ with increasing specimen mass are caused by increasing the absolute value of $\Delta G_{\text {nonc* }}^{P \rightarrow M}$ by increasing SCS. Incidentally, the present author and a coworker have recently shown that the logarithmic upward shifts of $T_{o}^{*}$ with increasing specimen mass observed in some shape memory alloys are ascribed to the logarithmic increase of SCS with increasing specimen mass. ${ }^{18}$ )

On the other hand, in SIMTs, externally applied stress is known to cause upward shifts of equilibrium temperature under stress $T_{o}(\sigma)$. It has been recently shown that the applied stress causes a larger increase of the elastic energy of a parent than that of a martensite, resulting in the upward shift of $T_{o}(\sigma) .{ }^{19)}$ This recent result indicates that the upward shifts of $T_{o}^{*}$ with increasing specimen size are similar to those of $T_{o}(\sigma)$ with increasing applied stress. Thus, we have found a clue to discussing the correspondence between TIMTs and SIMTs.

Thus, it has been proved that SCS and EBS play an important role in determining the characteristics of TIMTs. Details of the alterations of TIMT characteristics caused by varying SCS with specimen mass will be described elsewhere.

\section{Conclusions}

The present study is summarized as follows.

(1) The progress of TIMTs with changing temperature has been observed to have a sharp contrast to that of SIMTs under a constant stress. A paradox has been set up on the agreement between $M_{s}$ in TIMTs and temperatures $T_{s}^{L}(0)$ where the dependence of critical stress for inducing martensites $\sigma_{s}^{L}$ in SIMTs on temperature, $\mathrm{d} \sigma_{s}^{L} / \mathrm{d} T$, is extrapolated to stress zero.

(2) To resolve the paradox, a concept of inhomogeneous internal stress called shape change stress (SCS) has been introduced as an intensive variable. For martensite plates to form on cooling, the generation of SCS is required, because the plates must deform the surrounding untransformed parent. Increasing specimen size (mass) has been inferred to cause increasing SCS.

(3) By applying the Gibbs phase rule to MTs, the contrast in transformation behavior between TIMTs and SIMTs has been successfully explained. The progress of TIMTs by changing temperature has been attributed to inhomogeneous 
SCS, while that of SIMTs under a constant stress has been attributed to homogeneously applied external stress.

(4) The downward shifts of experimental equilibrium temperature $T_{o}^{*}=\left(M_{s}+A_{f}\right) / 2$ with decreasing specimen mass so far observed in TIMTs of some shape memory alloys have been attributed to decreasing SCS with decreasing specimen mass.

(5) Conceivably, no SCS is generated at all either in SIMTs or at the ultimate end of specimen size zero in TIMTs. This concept has rationalized the following observation on a monocrystalline $\mathrm{Cu}-13.4 \mathrm{Al}-4.2 \mathrm{Ni}$ (mass\%) alloy. That is, the variations of $M_{s}, M_{f}, A_{s}$ and $A_{f}$ with specimen mass have been extrapolated to specimen mass zero, resulting in $M_{s}^{\#}=M_{f}^{\#}$ and $A_{s}^{\#}=A_{f}^{\#}$. In addition, the former has coincided with $T^{L}(0)$ and the latter with $T^{U}(0)$. Thus, the paradox has been successfully resolved.

(6) It has been noted that SCS is important in discussing the correspondence between TIMTs and SIMTs.

\section{REFERENCES}

1) K. Otsuka, H. Sakamoto and K. Shimizu: Acta Metall. 27 (1979) 585601.

2) K. Otsuka, C. M. Wayman, K. Nakai, H. Sakamoto and K. Shimizu: Acta Metall. 24 (1976) 207-601.

3) S. Miyazaki, Y. Ohmi, K. Otsuka and Y. Suzuki: J. Phys. 43 (1982)
Suppl. 12. C4-255-C4-260.

4) H. Sakamoto: Proc. Intern'l. Conf. on Solid-Solid Phase Transformations '99 (JIMIC-3), (The Japan Institute of Metals, 1999) pp. 927-930.

5) H. Sakamoto: Journal of the Jpn. Copper and Brass Research Association, 39 (2000) 67-74.

6) H. Pops: Metall. Trans. 1 (1970) 251-258.

7) K. Otsuka and C. M. Wayman: Reviews on the Deformation Behavior of Materials, Freund Publ. House Ltd., Tel Aviv, Israel, 2(2) (1977) 81-172.

8) K. Otsuka and C. M. Wayman: Shape Memory Materials, (ed. by K. Otsuka and C. M. Wayman, Cambridge University Press, 1998, Cambridge, United Kingdom) pp. 27-48.

9) H. Sakamoto and K. Shimizu: Trans., JIM 25 (1984) 845-854.

10) H. Sakamoto, S. Sugimoto and T. Hara: Mater. Trans., JIM 39 (1998) 640-647.

11) R. J. Salzbrenner and M. Cohen: Acta Metall. 27 (1979) 739-748.

12) G. B. Stachowialk and P. G. MaCormick: Acta Metall. 36 (1981) 291297.

13) Y. M. Jin and G. J. Weng: Metall. Mater. Trans. A. 32A (2001) 305-313.

14) Y. Furuya, M. Matsumoto, H. Kimura, K. Aoki and T. Masumoto: Mater. Trans., JIM 31 (1990) 504-508.

15) H. Sakamoto, K. Meguro, A. Tanaka and A. Imai: J. Phys. IV 5 (1995) C8-581-C8-586.

16) Hi. Sakamoto, Ha. Sakamoto and S. Onai: Proc. Intern'l. Conf. on SolidSolid Phase Transformations '99 (JIMIC-3), (The Japan Institute of Metals, 1999) pp. 931-934.

17) S. Onai and H. Sakamoto: Mater. Sci. Forum, 327-328 (2000)393-396.

18) H. Sakamoto and S. Onai: Collected Abstracts of the 2001 Autumn Meeting of Japan Inst. Metals (2001) p. 77.

19) H. Sakamoto and S. Onai: Collected Abstracts of the 2001 Autumn Meeting of Japan Inst. Metals (2001) p. 76. 